Dermatologische Zeitschrift. 1935;71:I-IV

\title{
Contents, Vol. 71, 1935
}

\section{Inhaltsverzeichnis.}

Originalarbeiten.

Seite

Ackermann, Armín, Die Trichomonas vaginalis-Infektion 13 ?

Bartéls, Willy, Beitrag zur Frage der Wärmeurtikaria 201

Boas, Harold, und I. C. Neergaard, Kommt eine positive Wassermann-

Reaktion gelegentlich bei febrilen Lungenaffektione $\pi$ vor? 6

Chruschtschow, M., Über einen eigenartige $\pi$ intraepidermoidale $\pi$ adenoiden

Naevus in systematischer Ausbreitung am rechten Bein 19

Frohn, Wãh., und G. Schulze-Woüers, Erfahrungen über die Behandlung

der weibliche $\pi$ Go $\pi$ orrhoe mit Agesulf 16

Hruszek, H., Weitere Beobachtungen mit der Piiz-Miniatur-Kulturmethode 23 Jacoby, Guido,

Über die günstige Wírkung der mit Schwefelbäder $\pi$ kom-

binierten Quecksilberschmierkure $\pi 252$

Jahnel, Franz, und Berta Scharrer, Ein Beitrag zur Frage der Syphiiis-

empfindlichkeit im Tierreich, insbesondere verschiede $\pi$ er Mãusearten 1 Kitamura, S., Si $\pi$ d die

Erreger der Syphilis und der tropischen Framboesie

morphologisch võllig identisch? 61

Krumeich, B,, Spezifische Sensibilisierung bei der Gonorrhoe-Komplement-

Bindang 189

Leipner, S., Ergebnisse einer Trockenblutprobe auf Lupus 247

Leivin, I. M., Einiges über die Behandlung der díssetninierten Furunkulose

mit autogener und heterogener Vakzine

85

Leivin, M. M., Einige neue Befunde über Furunkel und Furunkulose.

(Hierzu Tafell)

MascMilleison, L. N., und L. A. Neraäoic, 33 Fälle von jadassohns

Granulosis rubra nasi 79

Melczer, N., Zur Morphologie des Birnennetzes (Golgi-Kopsch) der mensch-

lichen Talgdrüsenzellen

Neergaard J. C, siehe Boas, Harald.

Nékámjr., Ludwig, Über die eisophylaktische Funktion der Haut bei Syphilis 66

Neradow, L. A, siehe Maschkûleison, L. N.

Petrdcek, JSmil, Beitrag zur Ãtiologie der Melkerkлoten

71

Proppe, Albin, Zur Behandlung des Trippers der weiblichen Harnrõhre . 125 - und Hans Thurn,

Über die Compligon-Пautreaktion bei Trípperkranken 11

Bollier, A., Sonnen- und Hסhenluftbehandlung des Lupus 237

Scharrer, Berta, siehe Jahnel, Franz.

Schultze-Wolters, G., siehe Frohn, Wilh.

Spraße, H, Kasuistischer Beitrag zur Frage des Auftretens von Agranulo- 
zytose durch eine antisyphilitische Kur

Thurn, Hans, siehe Proppe, Albin.

Volk, B., Zur Biologic des Gonokokkus

graphisdier oder lehrbudimäßig zusammengefaßter Darstellung eigneten. Darauf ist es

zurüdtzuführen, daß der größte Teil der Büdier des Verlages auf seine Anregung ersdiienen ist und nidit lediglich in Übernahme an-gebotener Arbeiten. Dieser seiner hohen Auffassung von den Aufgaben ernes Verlegers entspraah es, daß er seinen Betrieb nidit in größtem organisatorisdien Stil aufzog, sondern auf einen klugbemessenen Umfang begrenzte.

Als Mensdi besaß der Verewigte viele sdiätzenswerte Eigensdiaften, und die hohe Aditung, deren er sidi erfreute, kam sdion bei seinem fünf-undzwanzigjährigen Verlagsjubiläum , in besonders wirkungsvoller Weise aber bei seinem JO. Geburtstage zum Ausdrudt. Mit Stolz erfüllte es ihn, wie unter Führung des hodibetagten, Jahrzehnte hindurdi in Freundsdiaft mit ihm verbundenen Geheimrats Prof. Martin eine große Zahl von be-deutenden Vertretern der Wissensdiaft und ärztlidien Praxis ihm in herzlidi gehaltenen anerkennenden Sdireiben huldigte. Aber audi dieser große Erfolg, der ihn hodi beglüdvte, spornte ihn nur zu neuen Taten an, und selbst den Verlust seiner innigst geliebten Lebensgefährtin, der ihn so sdiwer traf, überwand er in stiller Arbeit, soweit ein Mensdi in seinem Alter das vermag.

So bleibt das Bild S. Kargers uns unvergeßlidi als das eines guten und edlenMannes, der aus eigener Kraft einen weltbekannten angesehenen Verlag im Herzen Berlins sdiuf und der in der Gewißheit von uns gehen durfte, daß sein Sohn und seine Mitarbeiter alles daran setzen werden, um sein stolzes Lebenswerk weiter zu erhalten und zu fördern.

Die Schriftleiter der Zeitschriften des Verlages S. Karger
C. Behr
K. Bonhoeffer A. Czerny
A. Güttidi
E. Hoffmann

J. Meller

P. Morawitz L. Seitz F. Voltz

O.Wagener Joh. Zange

Pierersche Hofbgch $<$ jruckerei, Altenburg

IV Inhaltsverzeichnia.

Soit $\theta$

Sammelreferate.

Dermatitis, Ekzem und andere Allergosen. (Überbliek über dą Jaln· 1934.)

Von C. W. Beijerinck und J. A. Fo $3 / 4$ p»iers-Groningen

Erytheme, Herpesgruppe, Pemphigusgruppe, Blut- und Systemerkrankungen.

Von Dr. Albin Proppe-Düsseldorf 96

Dermatomykosen. Von Prof. Dr. P. W. Sclimiät-FrGihxirg i. Br 158

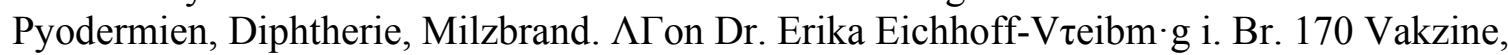
Variola, Alastrim, Melkerknoten, Scharlach, Masern, Varizellen,

Noma, Sodoku, Zoonosen. Von Dr. Erika Eichhoff-Freibm·g i. Br. . 174 Tuberkulose (1. April 1934 - 1. April 1935). Von Prof. Dr. Otto Bittrich-

Breslau 207

GummoseMykosen und verwandte Krankheiten (Sporotrichose, Blastomykose

usw.). Von 0. Grütz-Bonn a. Eh

Lepra. Von Prof. Dr. V. KlingmüUer-Kiel 219

Psoriasis, Lichenerkrankungen, Verhornungsanomalíen, Pigmentanomalien,

Nagel- und Haarerkrankungen , Erkrankungen der Talgdrüsen und

Schweißdrüsen. (Von Anfang 1934 bis Anfang 1935.) Von Dr. H. 0.

Zoos-Innsbruck 258

Geselischaftsberichte. 
Royal Society of Medicine. Section of Dermatology. Sitzung vom 18. Okt. 193453

Sitzung vom 15. November 1934

228

Sitzung vom 20. Dezember 1934

287

The North of England Dermatological Society. ïagung vom 11. Mai 1934

in Liverpool

55

- $\quad$ Sitzung vom 19. Oktober 1934 in Manchester

North British Dermatological Society. Sitzung vom 12. Dezember 1934 in

Edinburgh 115

The British Association of Dermatology and Syphilidologie. Jahrestagung in Newcastle-upon-Tyne am 6. und 7. Juli 1934

116

Société française de Derinatologie et de Syphiligraphie. Sitzungen vom

21. Juni und 12. Juli 1934

116

Sitzung vom 8. November $1934 \quad 230$

Sitzung vom 9. Dez. 1934288

Buchbesprechungen 57, 124, 234, 293

Personalien und Tagesnachrichten 60. 124, 180, 236, 296

Prof. Eugen Galewsky $\dagger$

Prof. Ernst Delbanco $\uparrow$

59

Wilhelm Kolle294 